\title{
Entrevista con Camila Villeruel: programas educativos y la familia en el museo - experiencias de la Fundación Proa
}

\section{DOI: http://dx.doi.org/10.5965/1984317815022019254}

Camila Villarruel tiene formación académica en Educación por el arte e Historia del Arte por la Universidad de Buenos Aires (UBA), en sus inicios como profesional trabajó en Centros de Día para personas con discapacidad y con problemas de adicciones. Desde hace más de diez años se desempeña en el desarrollo de programas para público escolar y familiar vinculados a la educación y los museos. Desde el año 2008 integra el equipo de educación de Fundación PROA, en Buenos Aires. En la actualidad se desempeña como uno de los referente de área y coordina las actividades vinculadas al público infantil y familiar.

1. Camila, agradezco su disponibilidad en colaborar con la revista Educação, Artes e Inclusão hablando sobre su trabajo en el equipo educativo de la Fundación Proa, en Buenos Aires. En la entrevista que Rosario García Martínez nos concedió (publicada en el volumen 15, n.1, de enero/marzo 20191), habló de las especificidades de Proa como espacio de arte contemporáneo y de las líneas generales del trabajo educativo. Me gustaría que usted pudiera adentrarse más específicamente en la cuestión de la relación de la Fundación con los niños y las familias, que es el trabajo que usted coordina. Le pido que para empezar nos hable un poco sobre cuáles son las principales proposiciones de Proa para los niños.

Camila Villaruel: En lo que se refiere a las escuelas, las distintas actividades que ofrecemos están organizadas en base a tres propuestas: tenemos lo que llamamos recorridos participativos (dejamos de usar el concepto "visita guiada"); después están las visitas con taller; y por último la articulación con el Museo Quinquela, que está aquí en frente y trabaja mucho la vinculación con la vida de Benito Quinquela [Benito Quinquela Martín (1890 - 1977) quien fue un reconocido pintor argentino nacido en el barrio de La Boca, donde se ubica Fundación Proa]. Estas son las tres principales actividades que se relacionan con las escuelas.

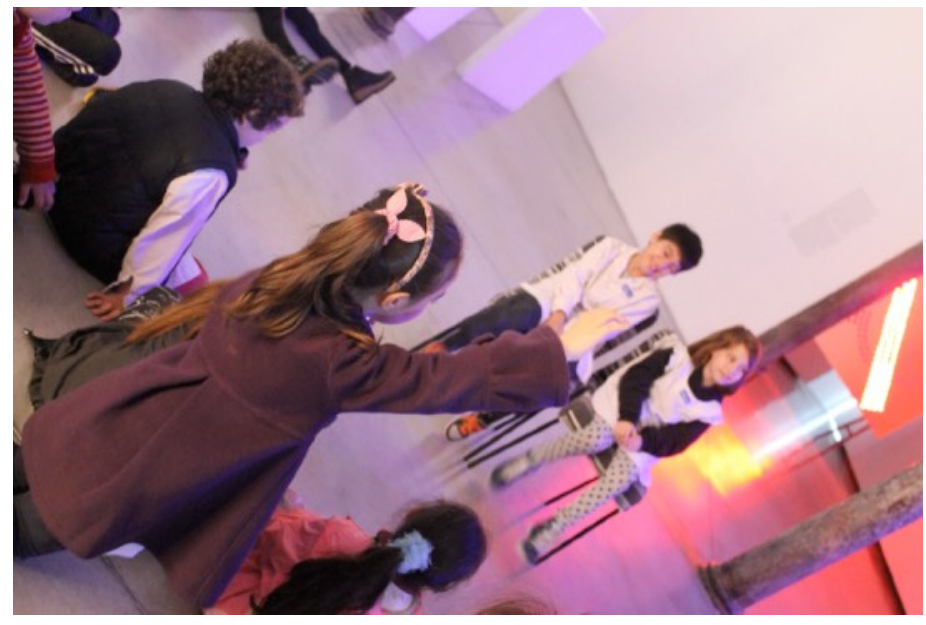

Actividad para escuelas.

Foto: equipo de educación de Proa

\footnotetext{
1 Disponible en el sitio web http://www.revistas.udesc.br/index.php/arteinclusao/article/view/14541
} 
Por otro lado contamos con un gran programa que es el de familias, en el cual trabajamos con propuestas para niños a partir de cuatro años, de cuatro a doce años, pero siempre en vínculo con el adulto. Lo que buscamos hacer con las actividades específicas que tenemos en este programa es que constantemente se esté generando un diálogo entre el adulto y el niño; no son actividades en las que el adulto vaya al café y el niño se quede participando, porque nuestra intención es que sea una experiencia compartida en la que ambos, de alguna forma, aprendan juntos y se vayan con preguntas y inquietudes vinculadas a la experiencia.

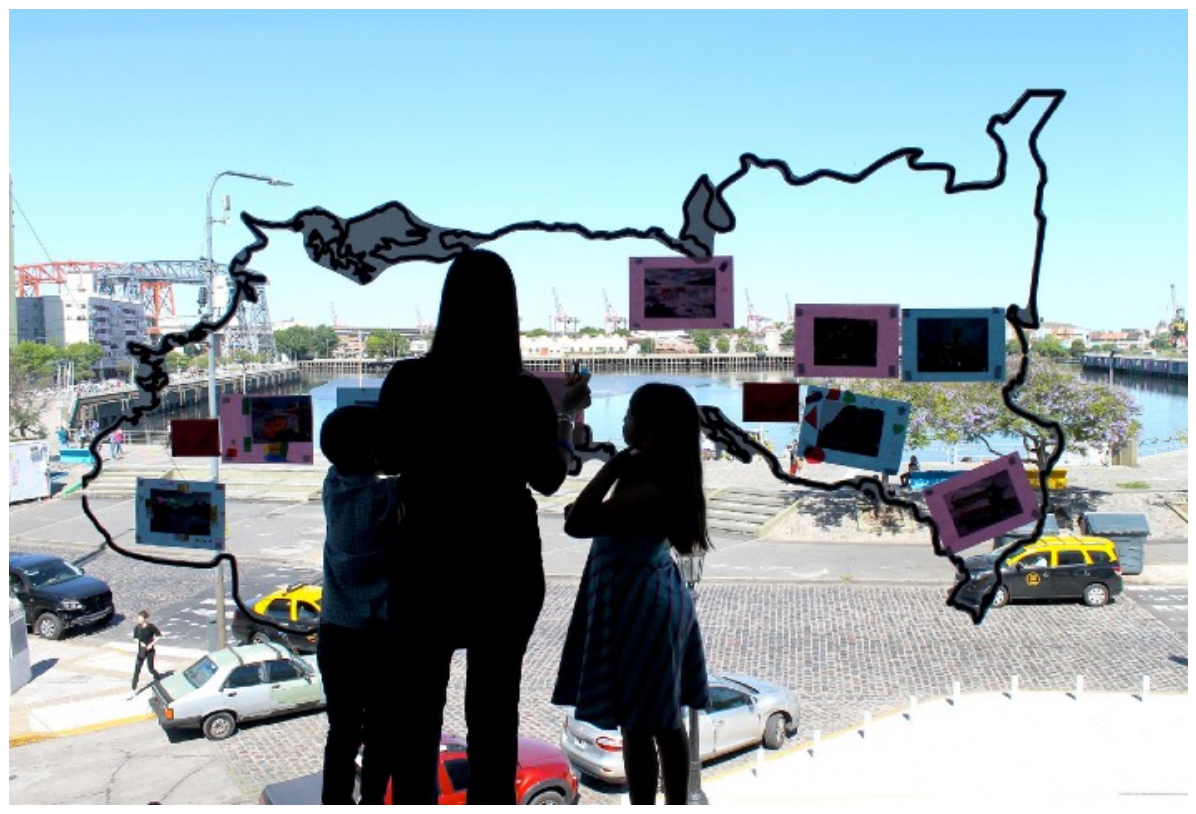

Actividad con familias

Foto: equipo de educación de Proa

2. ¿Cómo se efectúa el trabajo con las escuelas en las tres tipologías de actividad que usted citó: recorridos participativos, visitas con taller y articulación con el Museo Quinquela?.

Camila Villaruel: Los recorridos participativos son visitas en las salas de exhibición en donde se estabelece un dialogo entre los niños, el docente, el educador y las obras selecionadas; desde un aspecto lúdico, convocando al trabajo reflexivo y colectivo. En los reccoridos participativos ya hemos incorporado hace dos años que siempre haya materiales didácticos. Todos los materiales son producidos por el equipo de educación, y las actividades son diferentes para cada nivel educativo. En términos generales estos dispositivos didácticos ayudan a activar determinadas situaciones en el contexto de la visita. Las visitas con taller tienen un formato más tradicional, digamos, en el que primero se hace el recorrido en las salas y luego el taller en el espacio del auditorio y en el foyer. En los talleres se recuperan los conceptos trabajados en los recorridos. El objetivo de la asociación con el Museo Quinquela es realizar la articulación entre obras del patrimonio de Benito 
Quinquela con las obras que están expuestas en Fundación Proa, por medio de ejes conceptuales. Nos reunimos los dos equipos educativos y conversamos: "bueno, cómo podemos relacionar Calder [la exposición actualmente abierta en la Fundación Proa es Alexandre Calder: Teatro de Encuentros] con el acervo del Museo Quinquela?". Entonces, seleccionamos tres ejes distintos y empezamos a vincular esos ejes a determinadas metas. En general hacemos hojas de ruta, que son recursos para el grupo que abordan tanto el Museo Quinquela como la Fundación Proa, entonces es como una única visita que se divide en dos: media hora en Proa y media hora en el Quinquela. Y este recurso une los dos recorridos. Los itinerarios siempre cambian, porque en Proa nuestras exposiciones son de corta duración.

\section{3. ¿Y en relación a las actividades con familias?}

Camila Villaruel: En el programa de familias tenemos dos actividades: las valijas didácticas, que son para uso en las salas expositivas, y las jornadas de familias, que ocurren mensualmente, siempre en el segundo sábado de cada mes.
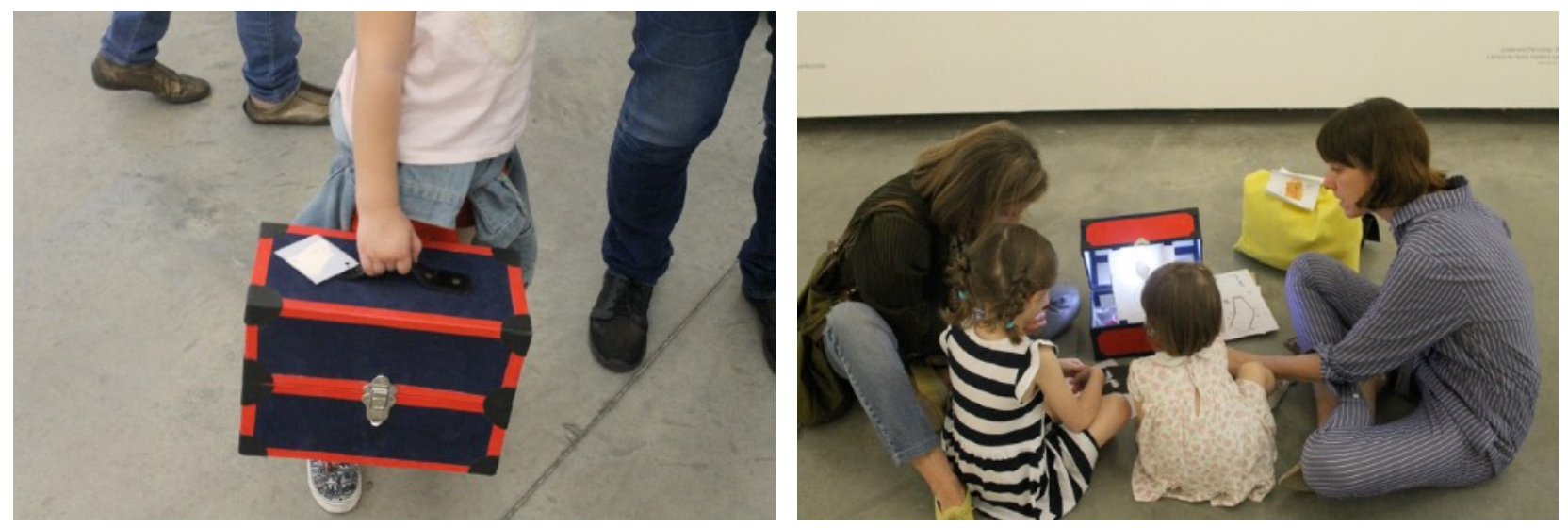

Familias interactuando con las valijas didácticas.

Foto: equipo de educación de Proa

El programa jornadas de familias tiene un año y medio, más o menos, y fue creciendo: hoy recibimos hasta 150 personas por día. Son cuatro talleres, hay atividades de las 14:30 a las 18:00 - dependiendo de la hora en que la familia llegue, va a una u otra, no es necesario que participe de todas - y nuestra intención es proporcionar distintas experiencias, en diferentes espacios, con un eje común. En el último encuentro el eje fue "Esto es una obra?", la idea era trabajar el objeto y sus distintas posibilidades de apropiación y de resignificación, digamos. Entonces había desde la posibilidad de hacer un ready-made hasta hacer intervenciones a partir de un objeto que trajeron: el taller se llamaba El museo que imagino y en él los niños escogían un espacio para su obra ... imagine a los niños encantados disponiendo los objetos en el espacio ... Otra actividad se llamaba Laboratorio de objetos encontrados, tenían que ir a buscar objetos que estaban escondidos en 
una parte del museo, con una maleta, y luego tenían que producir una obra-intervención: había un espacio y debían producir una instalación con los materiales que disponían en la maleta. Había también un teatro de sombras, una pieza que nosotros mismos creamos y que estamos usando para los recorridos participativos con educación inicial, y en los ventanales había una intervención en la que ellos eran invitados a usar binoculares, captar algún objeto a distancia y representarlo en la ventana usando vinilo. Ellos hicieron cosas maravillosas.

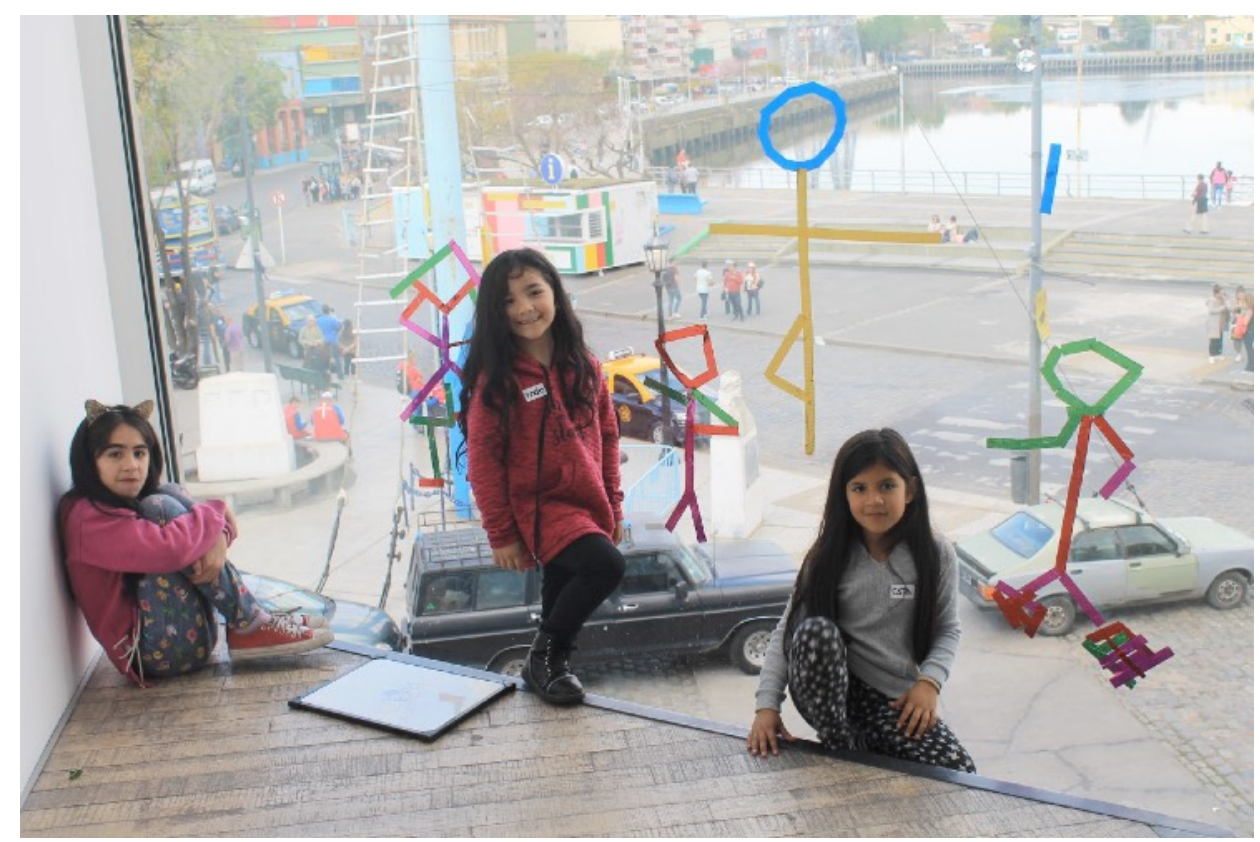

Actividad para familias.

Foto: equipo de educación de Proa.

Un diferencial del programa con familias es la posibilidad de una activación de todo el espacio de Fundación Proa, que no siempre es posible con los grupos de escuelas. Con las familias hacemos intervenciones en toda la parte del foyer. Intervinimos mucho en los ventanales, desde una pregunta pegada con vinilo hasta ... no sé, de todo; trabajamos con tecnología en el auditorio...

\section{4. ¿Cuáles son los ejes principales que fundamentan el trabajo con los niños en la Fun- dación Proa?}

Camila Villaruel: Los ejes principales para el trabajo con niños en Proa se relacionan menos con el "aprender" desde la perspectiva de que el conocimiento es transmitido al sujeto, y más con la idea de que es desde la experiencia que se adquiere el conocimiento. Lo que pretendemos en Proa es proporcionar una experiencia que sea distinta de las demás, por lo que no trabajamos tanto en una perspectiva escolar: si los niños están trabajando Calder en las escuelas de una determinada manera, procuramos trabajar desde otro punto de vista, para que las experiencias sean 
diferentes, para que no sea lo mismo que una clase. Lo mismo ocurre con las familias: si incorporamos, por ejemplo, tecnología, no vamos a hacer lo mismo que el niño tiene acceso con la tablet en su casa... Entonces lo que pretendemos es que la experiencia sea distinta. Otro eje de nuestro trabajo es el trabajo colectivo. Siempre son trabajos en equipo, nunca trabajamos de forma individual. Nuestro objetivo no es que el niño produzca una obra solo. Siempre se trabaja en equipo porque se pretende que las cuestiones individuales de cada niño sean potenciadas por el equipo. En ese sentido trabajamos el tema del respeto, de la escucha del otro; un gran objetivo es fomentar el trabajo colaborativo, que es lo que vivimos como equipo de educación, siempre trabajamos de esa forma. Y otra cuestión, un tercer eje, es la intención de trabajar de manera interdisciplinaria. La forma depende de cada exposición, pero nunca quedamos puramente en lo visual y plástico. Trabajamos el cuerpo, trabajamos los sonidos, trabajamos el vínculo con los espacios ... esos temas están siempre presentes en las visitas. Es muy importante para nosotros que los niños tengan conciencia de que tienen un cuerpo y están en un espacio, o sea, que no sólo tienen dos ojos para ver. Creemos que esto genera una experiencia mucho más rica; y si es más rica, es mejor, porque queda en el pensamiento ... Otra cuestión importante, para cualquier nivel escolar y para el trabajo con las familias, es que salgan con preguntas. No tenemos la intención de responder nada aquí: lo que pretendemos es que salgan intrigados, con ganas de saber más, sea sobre Calder o sobre la propia vida, sobre cualquier cosa. Siempre dejar una pregunta, y una pregunta abierta, para que ellos hagan nuevas preguntas. Esto es lo que genera que la experiencia no se limite al espacio de la Fundación Proa, sino que trascienda los muros.

\section{En el grupo Educação, Artes e Inclusão defendemos una perspectiva que afirma que es función de la educación crear necesidades, crear deseos...}

Camila Villaruel: claro, totalmente. Con los años, con la experiencia, vamos conociendo cada vez más sobre los niños, y nos damos cuenta de que hay muchas maneras de activar el deseo en ellos. Si usted tiene las estrategias, el niño va a conectarse solo, el estímulo es por medio de los materiales, de una pregunta hecha por el educador... hay mil formas... y yo creo que nuestra formación tiene que ver con eso: de qué forma podemos estimular ese deseo, esa voluntad, en los niños. Más que hablar para que aprendan: para eso ya tenemos la escuela y otros contextos. Entonces eso pienso que es importante: siempre hay un aprendizaje, pero esto está ligado a la experiencia.

\section{Usted mencionó que el equipo educativo vive el trabajo de forma colaborativa. ¿Cuál es la composición del equipo y cómo se estructura el trabajo educativo?}

Camila Villaruel: El equipo de educación tiene dos coordinadoras, yo estoy vinculada a los niños y Rosario García Martínez a los adultos, y también tenemos la figura del educador asociado, son cinco educadoras que forman parte del equipo educativo de forma estable, eso es importante. 
Nuestra intención, como coordinadoras, es que estén constantemente capacitándose, es un aprendizaje constante para todos nosotros, entonces la gente va creciendo también dentro de su función, van creciendo como educadoras, porque se van envolviendo cada vez más en los diferentes programas. Cada una tiene un perfil diferente. Entonces Rosario y yo nunca trabajamos solas, aquí todas las propuestas son pensadas en equipo, después hay una coordinación, digamos que toma una decisión final, pero todo es pensado en equipo a partir de reuniones. Lo que llamamos período entremuestras, cuando no hay exposición abierta, es el período de trabajo más intenso. Lo primero que hacemos es una especie de brainstorm de lo que es la exposición, sacamos ideas y pasamos a definir las metas de comprensión para cada nivel educativo y para cada programa, ¿qué es lo que queremos que el visitante entienda de este artista? Hacemos una especie de recorte: tenemos este artista, ¿qué es lo que nos interesa trabajar? A partir de eso tenemos las metas de comprensión, y a partir de las metas, las actividades. Entonces todo está articulado, coordinado. $Y$ todo se trabaja en equipo. Cuando comenzamos una nueva propuesta, hacemos una nueva reunión, sacamos las ideas generales y proponemos las actividades; después cada una trabaja en una actividad, pero las ideas nunca salen de una sola cabeza. Esto es importante porque las educadoras no sólo están en las salas - cuidando las obras, interactuando con el público - sino que también son partícipes de la propuesta educativa. Esto es algo que distingue a Fundación Proa, casi ningún museo en Buenos Aires tiene la figura del educador trabajando de forma colaborativa en las diferentes áreas.

\section{7. ¿Cómo el equipo educativo de la Fundación PROA ve la relación de los niños con el arte contemporáneo?}

Camila Villaruel: Los niños son mucho menos desprejuiciados que los adultos, al principio. No es que tengamos que pensar por comparación, pero la primera idea que me viene es que cuesta mucho menos que los niños entiendan por qué, por ejemplo, el mingitorio de Marcel Duchamp es una obra; a los adultos les cuesta mucho más. De hecho, nuestra primera exposición en Fundación Proa fue una muestra de Duchamp y los niños estaban muy involucrados con todo lo que era intervención en los objetos, como quien capta lo que está allí. Lo que yo veo en general es una relación muy sana entre los niños y las obras. Los adolescentes tienen más esa postura de "eso no es arte", pero en general la relación es buena. Depende mucho, de hecho, de cómo se trabaja. Te doy un ejemplo: una obra de [Cildo] Meireles que estuvo aquí en Proa, no recuerdo el nombre ... pero era una obra que estaba por todo el piso, tenía un hilo negro, era una intervención y en algún momento había como una pelota, pero era muy arte contemporáneo, ocupaba toda la sala la intervención con ese hilo negro. Los niños hicieron un ejercicio con el cuerpo por allí y luego ya estaban involucrados con la obra; no se hacen tanto la pregunta de si es arte o no es arte, por qué sí o por qué no, simplemente se entregan a la experiencia. Este cuestionamiento ocurre más con adolescentes. Pero con ellos lo que empezamos a hacer y que funciona muy bien 
es trabajar con intervenciones en el espacio. Por ejemplo, hubo hace poco una muestra que estaba vinculada a la fotografía, trabajaba la idea de la fotografía a través de un rasgo de la antropología visual. Dábamos a los adolescentes un corpus de obras, determinada cantidad, treinta fotografías por ejemplo, y lo primero que eran invitados a hacer era relacionar esas fotos con los temas que se les ocurrieran en el momento. De ese corpus de fotos, escogían 10 que estuvieran vinculadas a un tema, escogido por ellos. A partir de eso hacían una intervención en el espacio, podría ser en los ventanales, en la escalera... nosotros aquí en Proa no tenemos miedo de intervenir en el espacio. En fin, una intervención con un texto curatorial. Después cada grupo explicaba su trabajo. Este tipo de situación involucra a los adolescentes, situaciones en las que sean invitados a pensar, en las que sean protagonistas. El tema de "ni una a menos" apareció mucho, el tema del feminismo... Y eso es algo muy importante: hoy en día usted tiene la posibilidad de trabajar con un adolescente que es mucho más reflexivo que hace 25 años detrás, entonces es por ahí que usted tiene que estimularlo. Hicimos ese mismo taller con un grupo de docentes, ellos hicieron una intervención increíble por toda la escalera. La apropiación del espacio es muy importante, tanto para un niño pequeño como para un adolescente y un adulto. Entonces esa es una cuestión clave: no que se sientan en casa, porque no es una casa, sino que sientan que pueden ser partícipes activos, esa es la clave. En ese sentido, se puede mostrar a los visitantes cualquier obra que al principio no entienden, basta que se tengan estrategias para que se involucren en una experiencia comprensible para ellos - por qué a veces la obra es incomprensible... un limón en la pared... y por lo tanto es difícil, pero en general lo que hacemos es eso: "¿a qué idea te remite ese limón?" Y a partir de ahí vas a mediando ... Hicimos mucho esto en la exposición de Yves Klein que hubo aquí. La primera cosa que trabajamos era la percepción del color en el auditorio. Todo oscuro, proyectaba azul, púrpura y amarillo, y la primera invitación era que escribieran cuál era su primer pensamiento para cada uno de esos colores. De esa forma, usted accede por otro lado, digamos, y a partir de ahí consigue trabajar aspectos del artista. Intentamos salir un poco del artista también, crear una experiencia que vaya un poco más allá.

\section{Hablaste un poco sobre las especificidades de los adolescentes. ¿Y los niños pequeños, cuales son las especificidades del trabajo con ellos?}

Camila Villaruel: Lo principal con los niños pequeños es el trabajo con los materiales didácticos. Con la Educación Inicial - recibimos niños de 3 a 5 años, pero los grupos de tres años son raros, generalmente son niños de cuatro y cinco años - siempre tenemos algún material que pueda ser tocado, manipulado, porque en general en las exposiciones no se puede tocar nada. Lo principal es generar un interés desde lo visual, pero con materiales que puedan tener una conexión más clara con el niño pequeño. No trabajamos en equipos, porque no es adecuado, ellos todavía están muy individualizados, no saben aún trabajar en equipo. Y siempre pensamos materiales que llamen su atención. En el caso de la exposición actual, hay una maleta grande de la que salen per- 
sonajes, sale una carta grande escrita por Calder para los niños, todo es muy colorido ... Y las visitas terminan con un teatro de sombras dentro de la última sala de la exposición. Aprendimos con los años que debe haber espacios en los que los niños no tengan solo que escuchar, sino que también se generen espacios de participación. Por ejemplo, hubo aquí en Proa una muestra que se llamó RAQS Media Collective: es posible porque es posible. Era un grupo de arte muy contemporáneo y habia un escritorio en que se hacían preguntas, y la obra respondía. Usted se sentaba como espectador, preguntaba, y había una persona del otro lado que de acuerdo con la pregunta escogía una respuesta. Bueno, hicimos esa dinámica con los niños de cuatro años, usted no tiene idea de las preguntas que hacían, fue genial ... a ellos les gusta participar, más que estar paraditos escuchando ...

\section{El cuerpo para ellos es muy importante...}

Camila Villaruel: sí, jes muy importante! En los recorridos para nivel primario se trabaja mucho el vínculo del cuerpo con el espacio, el trabajo en equipo y la activación de otros sentidos más allá del visual: hacemos muchas cosas como que cierren los ojos, que miren a las obras de diferentes puntos de vista, que se acuesten para mirar... de sacarlos un poco de esa perspectiva más tradicional. El material pedagógico también funciona mucho con esta edad. Por ejemplo, en la primera sala de la exposición de Calder hacemos un ejercicio con unas pizarras pequeñas, en las que tienen dibujar a un compañero con sólo dos rasgos. Son pequeñas dinámicas en el espacio expositivo en el que se trabaja con distintas situaciones. Y lo más importante es eso: trabajar mucho la cuestión del cuerpo, del movimiento, de los diferentes sentidos. El trabajo varía de exposición a la exposición, pero esa es la idea.

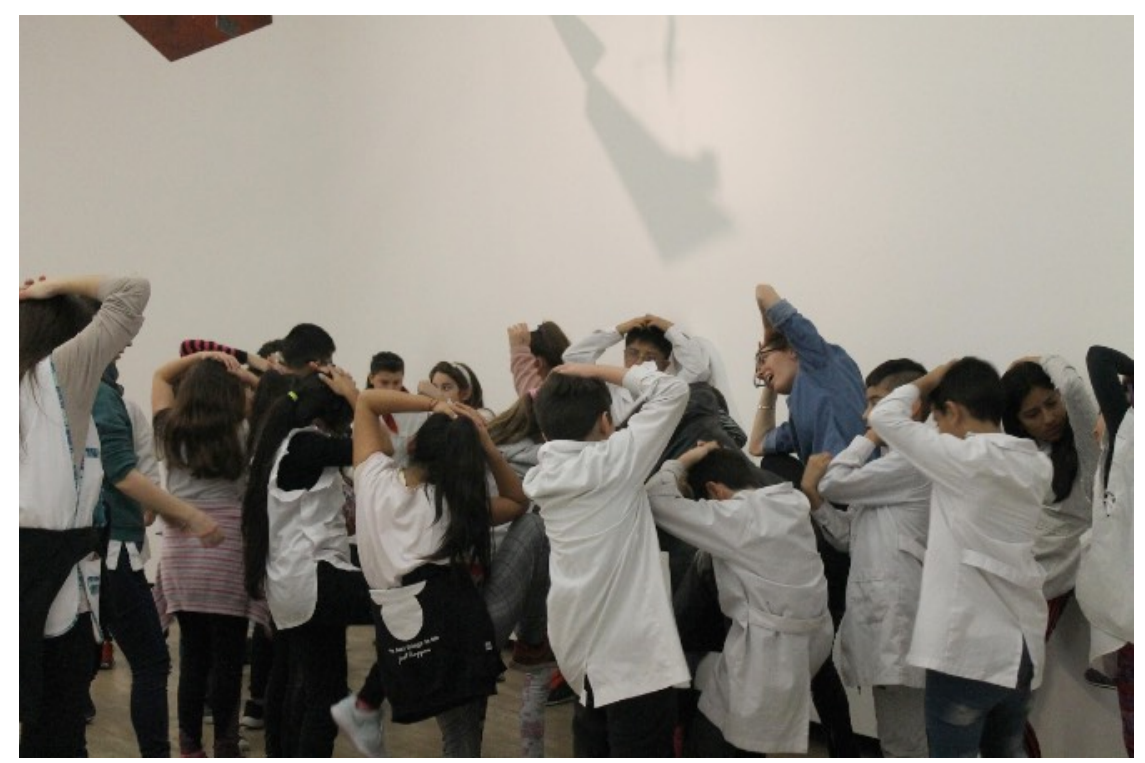

Actividad com escuelas.

Foto: equipo de educación de Proa 
Entrevista concedida a Flora Bazzo Schmidt en Fundación Proa, en Buenos Aires (Argentina), el 28 de septiembre de 2018.

Flora Bazzo Schmidt estudió pedagogía en la Universidad Federal de Santa Catarina y actúa en el Museo de Arqueología y Etnología Profesor Oswaldo Rodrigues Cabral, de la misma universidad. Actualmente hace su máster en Educación en la Universidad del Estado de Santa Catarina, bajo la dirección de la profesora María Cristina da Rosa Fonseca da Silva. Integra el grupo de investigación Educação, Artes e Inclusão y su revista académica. 